\title{
Produção científica de docentes de uma universidade estadual pública: 0 caso da Universidade Estadual da Paraíba
}

\section{Millena Nunes Dantas ${ }^{1}$, Anna Karolina Martins Borges², José Etham de Lucena Barbosa ${ }^{3}$, Sérgio de Faria Lopes ${ }^{2,3}$ e Rômulo Romeu Nóbrega Alves ${ }^{2,3}$}

\author{
${ }^{1}$ Universidade Estadual da Paraíba. Departamento de Ciências e Tecnologia. \\ Programa de Pós-Graduação em Ciência e Tecnologia Ambiental. Av. das Baraúnas, \\ 351. Campus Universitário. Bodocongó. Campina Grande-PB, Brasil (CEP \\ 58429-500). E-mail: millenanunesd@hotmail.com. \\ ${ }^{2}$ Universidade Estadual da Paraíba. Programa de Pós-Graduação em Etnobiologia e \\ Conservação da Natureza. Av. das Baraúnas, 351. Campus Universitário. \\ Bodocongó. Campina Grande-PB, Brasil (CEP 58429-500). \\ ${ }^{3}$ Universidade Estadual da Paraíba. Departamento de Biologia. Programa de \\ Pós-Graduação em Ecologia e Conservação. Av. das Baraúnas, 351. Campus \\ Universitário. Bodocongó. Campina Grande-PB, Brasil (CEP 58429-500).
}

Resumo. Este trabalho avalia indicadores de produtividade científica do corpo docente da Universidade Estadual da Paraíba (UEPB), entre os anos de 2011-2015. Um total de 911 currículos Lattes foram avaliados quanto ao número de publicações de livros, capítulos de livros, artigos, fomento (bolsistas PQ), título acadêmico, participação em programas de pós-graduação (PPG) e a soma de orientações concluídas a discentes de graduação e pós-graduação. Contabilizou-se um total de 3.816 artigos, 383 livros e 1.257 capítulos de livros. 55,8\% dos artigos foram publicados pelos docentes integrantes da pós-graduação. 14 docentes são detentores da bolsa produtividade CNPq. 290 docentes não tiveram nenhuma publicação científica no período analisado. Os pesquisadores das áreas das Ciências da Saúde e Ciências Biológicas apresentaram uma maior produção científica quanto à publicação de artigos em periódicos. As áreas de Ciências Humanas e Ciências Sociais Aplicadas tiveram a maioria de suas publicações associadas a livros e capítulos de livro em editoras nacionais. 0 maior número de publicações em revistas indexadas em bases internacionais está associado a docentes vinculados aos Departamentos de Biologia e de Enfermagem. Constata-se que a produção científica está concentrada em uma minoria de docentes, especialmente aqueles ligados a algum programa de pós-graduação, sobretudo os docentes pertencentes às áreas de Ciências Biológicas e Ciências da Saúde.

Recebido

$17 / 10 / 2020$

Aceito

18/01/2021

Disponível on line

$24 / 01 / 2021$

Publicado

30/04/2021

Acesso aberto

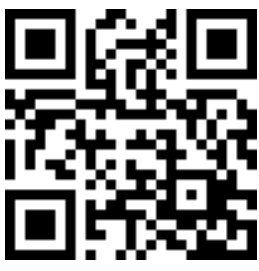

ISSN 2359-1412/RBGAS-2020-0180/2021/8/18/2/21

Rev. Bras. Gest. Amb. Sustent.

http://revista.ecogestaobrasil.net 
Palavras-chave: Cientometria; Publicação; Produtividade científica.

\begin{abstract}
Scientific production of teachers from a public state university: The case of the State University of Paraíba. This paper evaluates scientific productivity indicators of the faculty from the State University of Paraíba (UEPB), between the years 2011-2015. A total of 911 Lattes Curriculums were evaluated as for the numbers of book publications, book chapters, articles, fomentation (PQ scholarship), academic title, postgraduation programs participation (PPG) and the sum of orientations completed to graduates and postgraduates. An amount of 3816 articles, 383 books and 1257 book chapters were accounted. 14 teachers hold CNPq productivity scholarships. 290 did not have any scientific publications during the analyzed period. Researchers from Health Sciences and Biological Sciences presented greater scientific publication production as for article publication in periodicals. Human Sciences and Applied Social Sciences had the majority of their publications associated to books and book chapters in national publishers. The greater number of publications in indexed journals in international bases came from faculty associated to Biology and Nursing Departments. It finds that the scientific production is centered on a minority faculty, as a rule, those linked to some postgraduate program, especially those that belong to Biological Sciences and Health Sciences areas.
\end{abstract}

Keywords: Scientometry; Publication; Scientific productivity.
(1) 0000-0003-3609-892X Millena Nunes Dantas

D) 0000-0002-4951-1763 José Etham de Lucena Barbosa

D 0000-0003-4985-9927

Anna Karolina Martins Borges

D) 0000-0001-6472-6765 Sérgio de Faria Lopes

D) 0000-0001-6824-0797

Rômulo Romeu

Nóbrega Alves

\section{Introdução}

A produção científica brasileira tem evoluído em consequência, principalmente, da abertura de Programas de Pós-graduação em todo Brasil (Herculano e Norberto, 2011). Em 2019, informações do portal de indicadores bibliométricos Scimago (que utiliza dados da plataforma SCOPUS) indica que o Brasil ocupa a 14a posição no ranking de produção científica em número de documentos publicados (http://www.scimagojr.com). A análise da produção científica tem se tornado alvo de avaliações das carreiras acadêmicas por parte das agências financiadoras de pesquisa e das universidades, sendo fator importante, por exemplo, nas progressões funcionais dos docentes. Esta análise também, quando realizada pelas próprias universidades, tem o intuito de conhecer os pontos fortes e fracos das políticas de gerenciamento da instituição (Abramo e D’Angelo, 2015).

Publicação científica é parte integrante das atividades de um pesquisador. Alguns interesses adicionais levam os docentes a publicarem os resultados de suas pesquisas, dentre os quais a possibilidade de assegurar os financiamentos públicos (cada vez mais restritos) necessários para custeá-las, a garantia de sobrevivência no meio científico e o reconhecimento dos pares (Silva et al., 2003). Conforme Vessuri (1987), a ciência não publicada inexiste. Divulgá-la torna possível a mensuração do seu progresso, além de mostrar à sociedade a sua relevância. Nesse âmbito, tem sido intensificado análises associadas à cientometria, disciplina que faz uso de métricas capazes de quantificar o que vem sendo produzido pelos cientistas ou instituições de ensino (Carayol e Matt, 2006). As bases bibliográficas têm sido ferramentas cruciais para este tipo de avaliação (Herculano e 
Norberto, 2009), como indicadoras importante de qualidade dos veículos de publicação, e em nível internacional, sendo as principais bases a Web of Science (WoS) e o SCOPUS. Estas bases se destacam quanto ao alcance de uma ampla escala nas áreas científicas, pela sua influência internacional e por contabilizarem citações, que através de métricas como o índice- $h$ dos pesquisadores, possibilita a análise do impacto da produção dos mesmos. Adicionalmente, essas bases de dados têm contribuído na construção de outros indicadores, como o relatório do JCR (Journal Citation Reports), que avalia o impacto das revistas indexadas a Web of Science, sendo considerada uma das medidas mais usadas em todo mundo para análise da qualidade das revistas. De forma similar, a base SCOPUS também analisa o impacto de suas revistas através do Citescore.

Diversos trabalhos revelam que a concentração da produção científica está em uma minoria de pesquisadores vinculados às universidades/instituições de pesquisa; e que isso pode estar relacionado a questões individuais, como gênero, fomento, título acadêmico, carga docente (Diniz-Filho et al., 2016), e/ou a área de conhecimento a qual os pesquisadores pertencem. Com tudo isso, e ainda dependendo da escolha do docente quanto a forma de divulgar suas pesquisas, as diversas áreas irão apresentar indicadores de menor ou maior produtividade. Garvey e Griffth (1971) mostram que as escolhas de divulgação dos resultados de pesquisas divergem entre as áreas do saber e que isto se dá devido aos públicos-alvo distintos que exigem uma diversidade de canais que venham a atingi-los com maior eficiência.

Atualmente, a ciência de maior "impacto" vem sendo publicada em artigos periódicos indexados às bases renomadas (WOS e SCOPUS, por exemplo), tornando-a fidedigna, mas não consensual com àquela publicada em livros e capítulos. Velho (1997), em um de seus artigos, também levanta discussões acerca desse assunto, e reforça que nas áreas das ciências puras, exatas e naturais, é mais incidente a escolha de periódicos indexados em bases internacionais para publicação das pesquisas; enquanto que nas Ciências Sociais Aplicadas e nas Ciências Humanas existe uma tendência a escolher os canais regionais ou locais de comunicação, fazendo o uso de livros e capítulos, frente à necessidade de uma apresentação textual mais extensa para dar conta da complexidade das análises feitas. Não obstante, o grande número de periódicos disponíveis para a área de ciências sociais em nível internacional vai de encontro a essa visão. Somente o Social Sciences Citation Index (http://images.webofknowledge.com/WOKRS532MR24/help/ pt_BR/WOS/hp_database.html\#dsy36-TRS_social_sciences) um índice multidisciplinar para a literatura de periódicos de ciências sociais associado à Web of Science, abriga mais de 2.900 periódicos em 50 disciplinas das ciências sociais, além de indexar itens relevantes selecionados individualmente de mais de 3.500 periódicos técnicos e científicos entre os mais importantes do mundo.

No Brasil, a Plataforma Lattes, do CNPq, é um importante sistema de informação curricular que permite o registro da produção científica de pesquisadores, sendo utilizada principalmente para avaliações da produção científica de pesquisadores, áreas de pesquisa ou instituições. No presente estudo, utilizamos informações dessa plataforma para realizar uma análise da produção científica de docentes de uma Universidade Estadual da Paraíba. Considerando que a produtividade dos docentes variam tanto em função das áreas em que estes atuam, quanto de fatores em nível individual, no presente estudo, objetivou-se, (1) avaliar a produtividade científica dos docentes da Universidade Estadual da Paraíba (UEPB), (2) mensurar o desempenho dos docentes por áreas de conhecimento (ponderando as características nos diferentes campos da ciência), e (3) analisar a produção científica na instituição, por área de conhecimento, entre os anos 2011-2015.

Rev. Bras. Gest. Amb. Sustent., 2021, vol. 8, n. 18, p. 21-36. 


\section{Metodologia}

\section{A instituição}

A Universidade Estadual da Paraíba (UEPB) foi fundada em 1966. Com 54 anos de existência, a instituição possui oito Campi, sendo a sede localizada na cidade de Campina Grande (I), e os demais localizados nas cidades de Lagoa Seca (II), Guarabira (III), Catolé do Rocha (IV), João Pessoa (V), Monteiro (VI), Patos (VII) e Araruna (VIII).

Durante o período analisado por esta pesquisa, o corpo docente da instituição esteve composto por 950 docentes efetivos, distribuídos em 52 cursos de graduação (28 de licenciatura e 24 de bacharelado), quatro cursos técnicos de Pós-graduação à distância, 15 pós-graduações lato sensu e 20 programas de pós-graduação stricto sensu (mestrado e doutorado).

\section{Coleta de dados}

Inicialmente, foi acessado o site da Universidade Estadual da Paraíba e seus respectivos centros e departamentos para a coleta dos nomes dos docentes pertencentes a todos os campi da instituição. Entre os meses de outubro e dezembro de 2016 acessou-se a Plataforma Lattes, um repositório brasileiro online de currículos mantido pelo Conselho Nacional de Desenvolvimento Científico e Tecnológico (CNPq), a fim de acessar os dados dos currículos indexados, a partir do campo de "busca por pesquisadores", no qual foi digitado o nome completo de cada docente. Foram compiladas manualmente as informações referentes ao (1) gênero do(a) docente, (2) titulação, (3) carga docente, (4) detentor ou não de bolsa de produtividade do CNPq, (5) participação em programas de pós-graduação, (6) número de publicações de artigos (7), livros e (8) capítulos de livros nacionais e internacionais, e o (9) número de orientações concluídas direcionadas à estudantes de graduação, especialização, mestrado e doutorado entre os anos 2011-2015. Por fim, foram acessadas as bases internacionais WoS e SCOPUS, para a verificar a indexação das revistas nas quais os artigos publicados. Por meio dessas bases, também foi possível obter o índice- $h$ de cada docente. Esse índice, como proposto por Hirsch (2005), avalia a produção científica dos pesquisadores através da combinação da produtividade e o impacto da pesquisa.

Categorizou-se os docentes analisados em nove áreas de conhecimento, com base na classificação da Coordenação de Aperfeiçoamento de Pessoal de Nível Superior (CAPES, 2017), conforme disposto a seguir: Ciências Agrárias (CA), Ciências Biológicas (CB), Ciências Humanas (CH), Ciências da Saúde (CS), Ciências Sociais Aplicadas (CSA), Ciências Exatas e da Terra (CET), Engenharias (E), Linguística, Letras e Artes (LLA) e a Multidisciplinar (M).

Todos os dados foram organizados no software Microsoft $®$ Office Excel® 2007. Para observar as diferenças na produção científica entre os sexos (para artigos, livros, capítulos e a soma total), e entre as áreas de conhecimento, foi realizado o teste não paramétrico Kruskal-Wallis e a posteriori o teste Dunn's para identificar quais áreas, especificamente, apresentaram diferenças estatisticamente significativas. 0 teste de Kruskal-Wallis (KW) é um teste não paramétrico utilizado para comparar três ou mais populações de dados. Ele é usado para testar a hipótese nula de que todas as populações possuem funções de distribuição iguais contra a hipótese alternativa de que ao menos duas das populações possuem funções de distribuição diferentes. 0 teste de Kruskal-Wallis é o análogo ao teste $\mathrm{F}$ utilizado na ANOVA de 1 fator. Já o teste de Dunn analisa os pares específicos de amostras para dominância estocástica em testes post hoc.

Por fim, para detectar se houve diferenças significativas entre o gênero dos docentes para o número de publicações de artigos, livros, capítulos de livros e a soma total foi utilizado o teste Mann-Whitney. Este é um teste não paramétrico aplicado para duas amostras independentes. 0 teste Mann-Whitney é a versão da rotina de teste 
não-paramétrico de $t$ de Student. As análises foram efetuadas com auxílio do software PAST versão 3.16 (Hammer et al., 2001).

\section{Resultados}

A consulta à Plataforma Lattes (2016) possibilitou a extração das informações referentes a 911 docentes do quadro efetivo da UEPB. Os demais $(4,1 \%)$, não tinham cadastro de currículo na referida plataforma. A partir dos dados levantados constatamos que as áreas que possuíam um maior número de docentes foram Ciências Humanas, Ciências Sociais Aplicadas, Ciências da Saúde e Ciências Exatas e da Terra, respectivamente (ver Tabela 1 ).

Tabela 1. Número total de docentes; artigos publicados; artigos indexados às bases WoS e SCOPUS; supervisão concluída a discentes de graduação, especialização, mestrado e doutorado; produção de livros e capítulos nacionais e internacionais de acordo com as áreas de conhecimento.

\begin{tabular}{|l|c|c|c|c|c|c|c|c|c|c|}
\hline Produção & CA & CB & CH & CS & CSA & CET & E & LLA & M & Total \\
\hline Docentes & 43 & 57 & 200 & 162 & 168 & 147 & 29 & 98 & 7 & 911 \\
\hline Artigo & 251 & 566 & 478 & 1469 & 410 & 328 & 100 & 191 & 23 & 3816 \\
\hline Web of Science & 74 & 322 & 20 & 440 & 58 & 152 & 30 & 1 & 12 & 1109 \\
\hline SCOPUS & 84 & 357 & 29 & 499 & 64 & 150 & 33 & 1 & 10 & 1227 \\
\hline Livro Nacional & 2 & 20 & 115 & 23 & 82 & 32 & 5 & 85 & 0 & 364 \\
\hline Livro Internacional & 0 & 4 & 2 & 1 & 6 & 2 & 0 & 2 & 2 & 19 \\
\hline Capítulo Nacional & 20 & 67 & 393 & 122 & 216 & 76 & 13 & 254 & 1 & 1162 \\
\hline Capítulo Internacional & 2 & 28 & 14 & 15 & 18 & 15 & 2 & 1 & 0 & 95 \\
\hline TCC & 147 & 421 & 1385 & 810 & 1531 & 735 & 120 & 520 & 1 & 5670 \\
\hline TCC (Esp.) & 9 & 67 & 594 & 134 & 280 & 231 & 18 & 175 & 1 & 1509 \\
\hline Dissertação & 23 & 163 & 158 & 282 & 87 & 110 & 64 & 123 & 1 & 1011 \\
\hline Tese & 5 & 20 & 6 & 25 & 9 & 10 & 4 & 10 & 0 & 89 \\
\hline
\end{tabular}

CA = Ciências Agrárias; $\mathrm{CB}$ = Ciências Biológicas; $\mathrm{CH}=$ Ciências Humanas; $\mathrm{CS}$ = Ciências da Saúde; CSA = Ciências Sociais Aplicadas; CET = Ciências Exatas e da Terra; E = Engenharias; LLA = Linguística, Letras e Artes; M = Multidisciplinar.

A análise dos dados mostrou a prevalência de pesquisadores do sexo masculino no corpo docente das áreas Multidisciplinar (86\%), Ciências Agrárias (67\%), Exatas (59\%), Engenharias (55\%) e Biológicas (52\%), enquanto que nas áreas das Ciências da Saúde (65\%), Linguística, Letras e Artes (60\%), Ciências Humanas (58\%) e Ciências Sociais Aplicadas (53\%) prevaleceu a maior participação feminina. No geral, constatou-se uma proporção similar entre os docentes, por gênero, na UEPB, com a participação de 53,2\% de docentes mulheres contra 46,8\% de docentes homens. Não houve diferença significativa na produção científica total dos docentes de acordo com o gênero (ver Tabela 2), exceto na soma de capítulos de livros nacionais $(P=0,0033)$, situação em que houve um maior número de capítulos publicados $(\mathrm{n}=261 ; 36,7 \%)$ por parte do corpo docente feminino. 
Tabela 2. Número de docentes do sexo masculino e feminino. Valor P do teste Mann-Whitney entre os gêneros para o número de publicações de artigos, livros e capítulos de livros de cada grupo.

\begin{tabular}{|l|c|c|c|}
\hline \multirow{2}{*}{ Produção } & \multicolumn{2}{|c|}{ Gênero } & \multirow{2}{*}{ p-valor } \\
\cline { 2 - 3 } & Masculino & Feminino & \\
\hline Artigo & 2062 & 1754 & 0,725 \\
\hline Livro nacional & 189 & 175 & 0,784 \\
\hline Livro internacional & 10 & 9 & 0,790 \\
\hline Capítulo nacional & 450 & 711 & 0,003 \\
\hline Capítulo internacional & 58 & 37 & 0,377 \\
\hline Total & $\mathbf{2 7 6 9}$ & $\mathbf{2 6 8 6}$ & 2,679 \\
\hline
\end{tabular}

Constatou-se que apenas 14 pesquisadores da UEPB eram detentores da Bolsa Produtividade (PQ) com bolsa vigente no período da pesquisa, 13 do gênero masculino e vinculados às áreas das Ciências da Saúde $(\mathrm{n}=3)$, Ciências Biológicas $(\mathrm{n}=3)$, Engenharias $(\mathrm{n}=3)$, Ciências Exatas e da Terra $(\mathrm{n}=2)$, Ciências Sociais Aplicadas $(\mathrm{n}=1)$ e Ciências Humanas $(n=1)$. A única mulher bolsista $P Q$ atua na área da Saúde, especificamente no Curso de Enfermagem, no qual há uma maior concentração de mulheres.

A distribuição da produção docente de acordo com o tipo de publicação resultou em um total de 3.816 artigos, 383 livros e 1.257 capítulos de livro. Considerando a publicação em artigos científicos, os dados apontaram que os docentes com o maior número de artigos estavam ligados às áreas da Saúde (Odontologia: 766; Enfermagem: 410 e Farmácia: 212) e das Ciências Biológicas (Biologia: 566 artigos).

A área das Ciências Humanas responde pelo maior número de publicações de livros e capítulos de livros nacionais $(n=508 ; 33,3 \%)$, seguida da área Linguística, Letras e Artes $(n=339 ; 22,2 \%)$ e Ciências Sociais Aplicadas $(n=298 ; 19,5 \%)$. Esta última também detém a maior quantidade de livros internacionais publicados $(n=6 ; 31,6 \%)$, seguida de perto pela área de Ciências Biológicas, com quatro livros, apesar da área ser representada por apenas dois cursos. No entanto, quando se considera capítulos de livros internacionais, a maior parte desses $(\mathrm{n}=28 ; 29 \%)$ foram publicados pelos docentes das Ciências Biológicas (ver Figura 1). Destes, 20 capítulos foram publicados por um único pesquisador da área.

Os dados de supervisão a discentes de graduação, especialização, mestrado e doutorado, mostraram que os docentes dos cursos pertencentes às áreas das Ciências Sociais Aplicadas e das Ciências Humanas, apresentaram números mais elevados de orientação a alunos de graduação e especialização quando comparado às outras áreas de conhecimento (ver Figura 2). Em números absolutos essas duas áreas resultaram em um total de 1979 (27,6\%) e 1811(25,2\%) orientações de TCC defendidas, respectivamente. Em relação a supervisão de alunos de mestrado, as áreas das Ciências da Saúde e das Biológicas respondem pelos maiores percentuais, com total de 27,9\% ( $\mathrm{n}=282$ ) e $16,1 \%$ $(n=163)$ dissertações, respectivamente. No que se refere a orientação à alunos de doutorado, também, as áreas das Ciências da Saúde e das Biológicas lideram, apresentado um percentual de $28,1 \%(n=25)$ e $22,47 \%(n=20)$ de teses defendidas.

No levantamento de dados referente ao número de artigos publicados em periódicos indexados às bases SCOPUS e WoS foram computados $1.227(24,1 \%)$ publicações em revistas indexadas à primeira, e 1.109 (22,2\%) em revistas indexadas à segunda. 


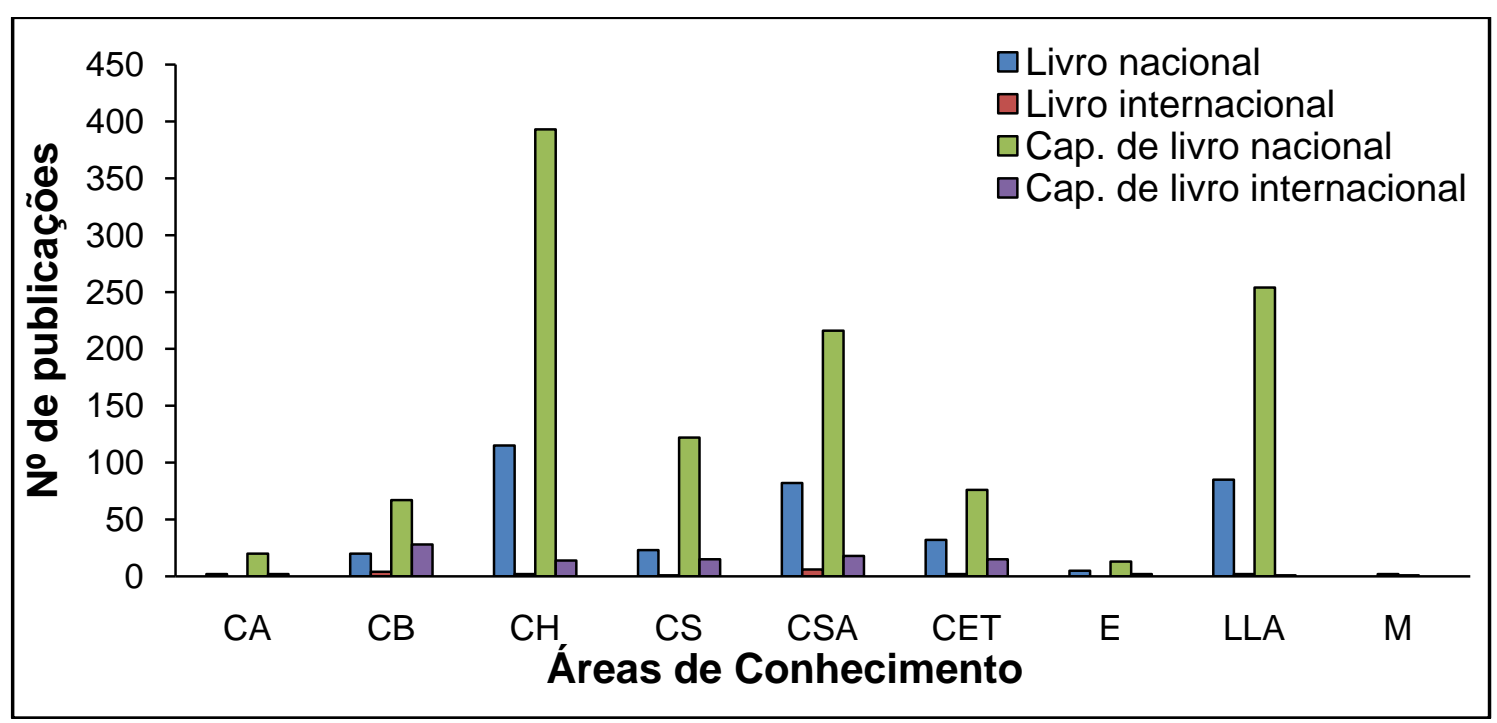

Figura 1. Número total de publicações de livros e capítulos de livros nacionais e internacionais (As abreviações estão contidas na Tabela 1). CA = Ciências Agrárias; $\mathrm{CB}$ = Ciências Biológicas; $\mathrm{CH}=$ Ciências Humanas; $\mathrm{CS}$ = Ciências da Saúde; CSA = Ciências Sociais Aplicadas; CET = Ciências Exatas e da Terra; E = Engenharias; LLA = Linguística, Letras e Artes; $\mathrm{M}=$ Multidisciplinar.

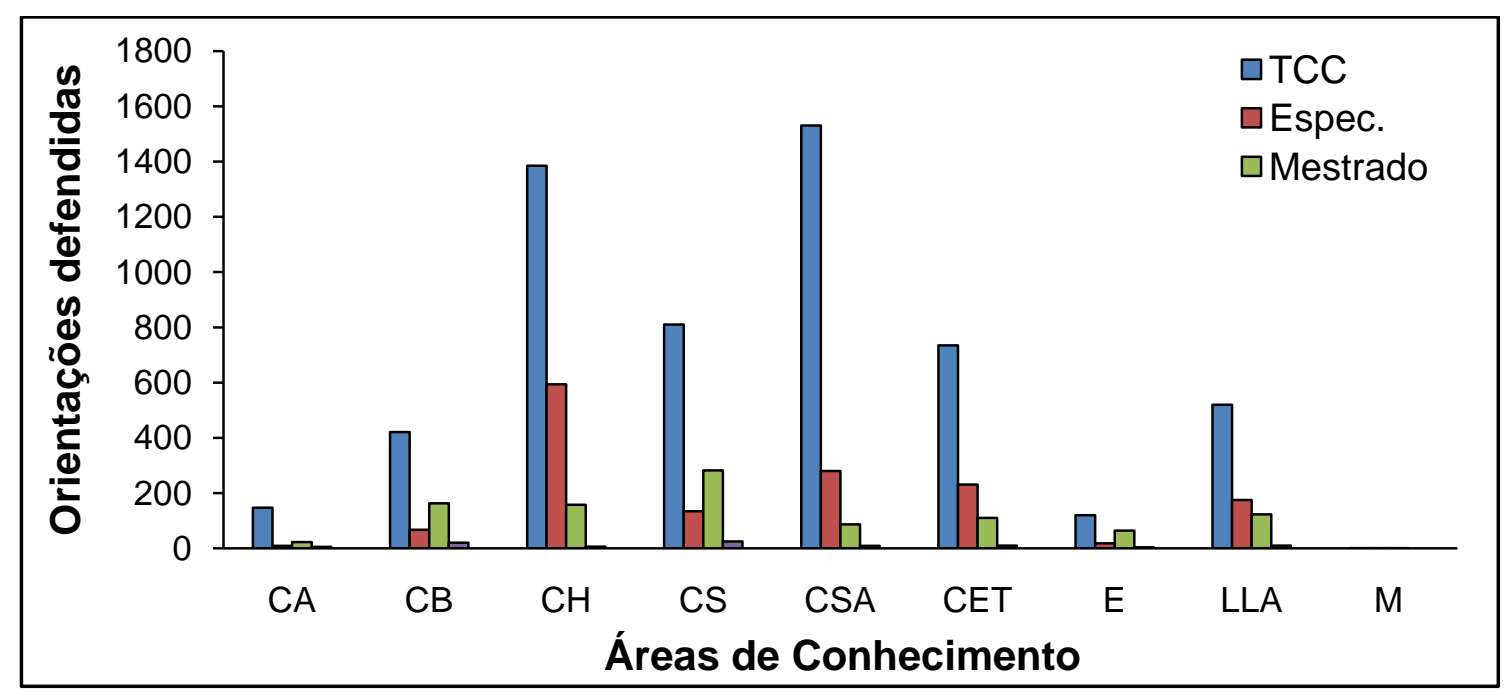

Figura 2. Orientações defendidas pelos discentes de Graduação e Pós-graduação, supervisionados pelos docentes da UEPB. CA = Ciências Agrárias; $\mathrm{CB}=$ Ciências Biológicas; $\mathrm{CH}$ = Ciências Humanas; CS = Ciências da Saúde; CSA = Ciências Sociais Aplicadas; CET = Ciências Exatas e da Terra; E = Engenharias; LLA = Linguística, Letras e Artes; $\mathrm{M}=$ Multidisciplinar.

As áreas com maior número de artigos publicados em periódicos indexados foram as Ciências Biológicas e as Ciências da Saúde. A primeira teve $56 \%$ de suas pesquisas publicadas em revistas indexadas à base $W o S$, enquanto a segunda teve $30 \%$ de indexações nessa mesma base. A área das Biológicas apresentou $63,1 \%$ de sua produção de artigos indexada ao SCOPUS e Ciências da Saúde 34\%. Outro fato relevante é que a área das Ciências da Saúde é representada na UEPB por seis Departamentos (Educação Física, Enfermagem, Farmácia, Fisioterapia e dois de Odontologia), enquanto que a área das 
Ciências Biológicas possui apenas dois departamentos de Biologia, revelando que, proporcionalmente os docentes do Departamento de Biologia respondem pela maior produção científica da UEPB por docente, mesmo com um menor número de cursos e consequentemente de docentes (162 de Ciências da Saúde e 57 Ciências Biológicas). A área Multidisciplinar teve $52 \%$ de suas pesquisas indexadas a $W o S$ e $43 \%$ ao SCOPUS. A área de Ciências Agrárias teve 29\% de suas pesquisas indexadas à WoS e 33\% ao SCOPUS. A área de Linguística, Letras e Artes publicou 191 artigos e apenas um indexado em cada uma dessas bases. A área de Ciências Exatas e da Terra teve pouco mais de $45 \%$ de suas pesquisas publicadas em revistas indexadas à WoS e SCOPUS. Embora a Área das Ciências Humanas seja a que possui um maior número de docentes $(n=200)$, seguida da área das Ciências Sociais Aplicadas ( $\mathrm{n}=168)$, a produção cientifica por parte desses docentes quanto à publicação de artigos (indexados ou não às bases WoS e SCOPUS) é baixa.

Foi constatada a presença de pesquisadores que apresentam uma maior produtividade vinculada às áreas $\mathrm{CB}$ e CS quando comparada com as outras áreas de conhecimento (ver Figura 3). Estas áreas apresentaram diferenças significativas $(\mathrm{P}<0,05)$ quanto ao número de publicação em periódicos indexados.

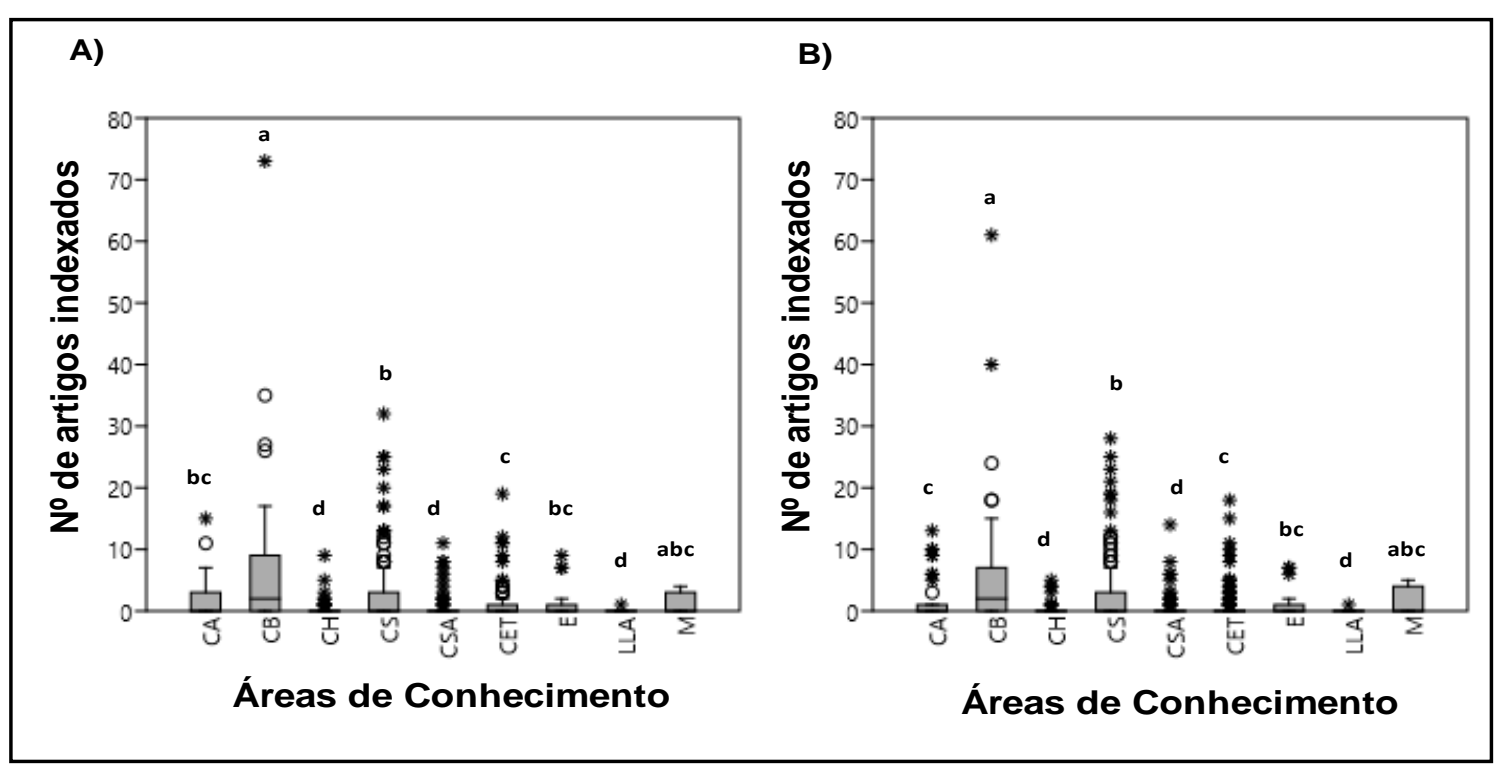

Figura 3. Número total de publicações de artigos periódicos dos docentes da UEPB indexados às bases (A) SCOPUS e (B) WoS, de acordo com as áreas de conhecimento. Os asteriscos e os círculos representam os outliers. Letras distintas mostram diferenças significativas $(\mathrm{P}<0,05)$. CA $=$ Ciências Agrárias; $\mathrm{CB}=$ Ciências Biológicas; $\mathrm{CH}=$ Ciências Humanas; $\mathrm{CS}=$ Ciências da Saúde; $\mathrm{CS}$. A = Ciências Sociais Aplicadas; CET = Ciências Exatas e da Terra; E = Engenharias; LLA = Linguística, Letras e Artes; $\mathrm{M}=$ Multidisciplinar.

Os dados de índice- $h$ dos pesquisadores, obtidos nas bases de dados SCOPUS e WoS nos forneceu uma estimativa de impacto da produção científica em nível individual. A partir da Tabela 3 verifica-se que apenas $19 \%$ dos docentes apresentaram índice- $h \geq 1$ pelo $W o S$ e $18 \%$ pelo SCOPUS. 
Tabela 3. Número de docentes da UEPB em relação aos respectivos índices- $h$ via $W o S$ e SCOPUS.

\begin{tabular}{|l|c|c|}
\hline Índice- $\boldsymbol{h}$ & WoS & SCOPUS \\
\hline 1 & 67 & 41 \\
\hline 2 a 5 & 63 & 90 \\
\hline 6 a 10 & 17 & 29 \\
\hline Maior que 10 & 2 & 6 \\
\hline Total & $\mathbf{1 4 9}$ & $\mathbf{1 6 6}$ \\
\hline
\end{tabular}

Os resultados mostraram que a área das Ciências Biológicas apresentou as médias mais elevadas para os índices-h, tanto no SCOPUS quanto pela WoS (ver Figura 4). Um total de $710(77,94 \%)$ docentes não apresentam índice- $h$ em nenhuma das duas bases. Do total de docentes $(n=166)$ que apresentam índice- $h$, houve variação de 1 a 31 no SCOPUS e de 1 a 25 na $W o S$. A média de índice- $h$ foi de 3,6 pelo SCOPUS e 2,80 pelo WoS. Os valores da mediana foram de 3 e 2 , respectivamente. Os maiores valores de índice- $h$ compilados (em ordem decrescente) foram observados entre os docentes dos departamentos de biologia (um docente com $\mathrm{h}=31$ pelo SCOPUS e h $=25$ pelo $W o S$ ) e de enfermagem (um docente com $\mathrm{h}=17$ pelo SCOPUS e $\mathrm{h}=2$ pelo WoS, e uma docente $\operatorname{com} \mathrm{h}=14$ pelo SCOPUS e $\mathrm{h}=12$ pelo $W o S$ ). Em contrapartida, a maioria dos docentes vinculados às áreas das Ciências Humanas $(\mathrm{n}=188)$, Sociais Aplicadas $(\mathrm{n}=159)$ e Linguística, Letras e Artes $(\mathrm{n}=98)$ não tinham índice- $h$. Aqueles que tinham, os valores eram baixos, reflexo da pouca produção de artigos publicados em periódicos indexados às bases em questão.

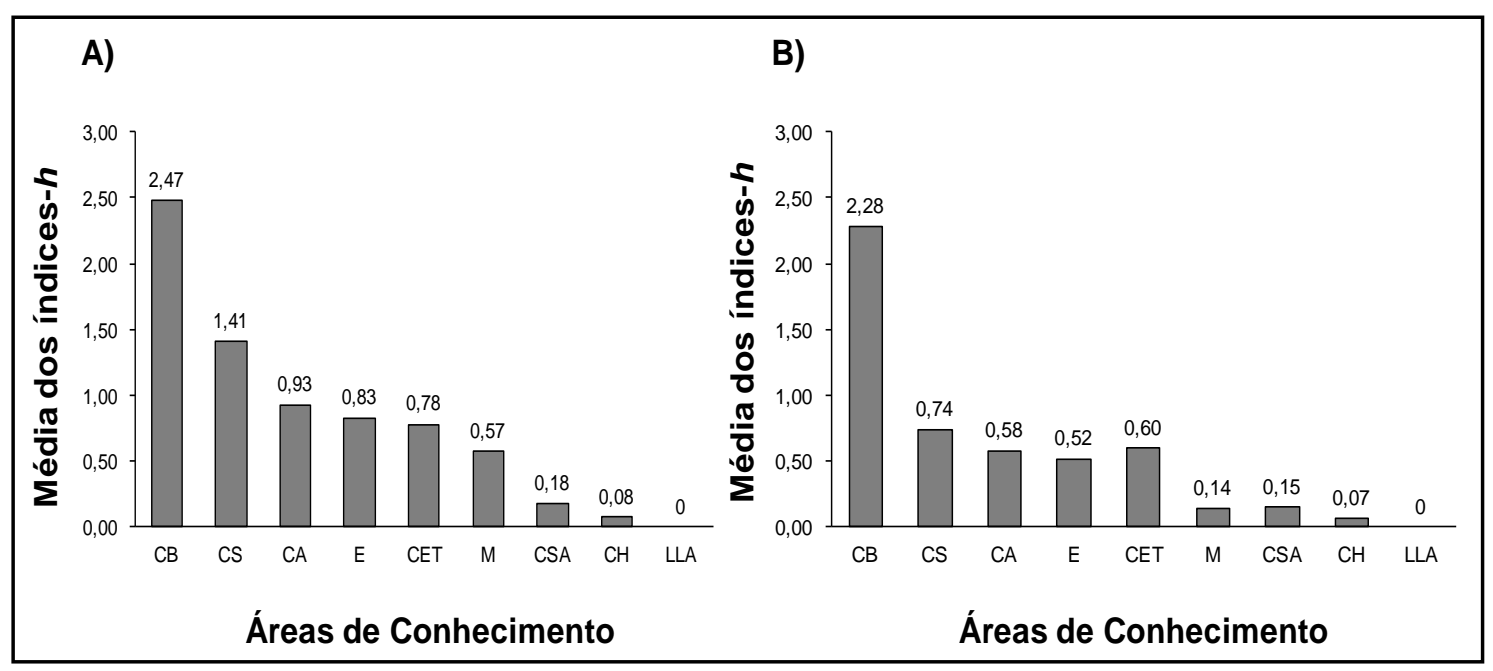

Figura 4. Média dos índices- $h$ pelas bases (A) SCOPUS e (B) WoS, dos docentes da UEPB. CA = Ciências Agrárias; $\mathrm{CB}=$ Ciências Biológicas; $\mathrm{CH}$ = Ciências Humanas; $\mathrm{CS}$ = Ciências da Saúde; $\mathrm{CS}$. A = Ciências Sociais Aplicadas; CET = Ciências Exatas e da Terra; E = Engenharias; LLA = Linguística, Letras e Artes; $\mathrm{M}=$ Multidisciplinar.

As pós-graduações ofertadas pela UEPB, ligadas aos seus respectivos centros, estão elencadas na Tabela 4. 0 total de 191 (21\%) docentes está vinculado aos programas de Pós-graduação, sendo a maior parte da produção da UEPB $(55,8 \%)$ advinda desses docentes. Entre os anos 2011-2015, 245 novos docentes adquiriram o título de doutor, mas apenas uma parcela deles $(n=74)$ contribuiu na produção de artigos periódicos no período avaliado. 
Tabela 4. Programas de Pós-graduação (PPG) ofertados pela UEPB, vinculados aos seus respectivos Centros.

\begin{tabular}{|l|c|}
\hline Programas de Pós-Graduação & Centros \\
\hline Ciências Agrárias - PPGCA & $*$ \\
\hline Etnobiologia e Conservação da Natureza - PPGETNO & CCBS \\
\hline Ecologia e Conservação - PPGEC & CEDS \\
\hline Desenvolvimento Regional - PPGDR & $*$ \\
\hline Formação de Professores - PPGPFP & CCBS \\
\hline Enfermagem - PPGE & CCT \\
\hline Ciência e Tecnologia Ambiental - PPGCTA & CCBS \\
\hline Ciências Farmacêuticas - PPGCF & CCT \\
\hline Ensino de Física - PPGPEF & CH \\
\hline Profissional em Letras - PROFLETRAS & CEDUC \\
\hline Literatura e Interculturalidade - PPGLI & CCT \\
\hline Ensino de Ciências e Educação Matemática - PPGECEM & CCBS \\
\hline Odontologia - PPGO & CCBS \\
\hline Psicologia da Saúde - PPGPS & CCT \\
\hline Química - PPGQ & CCSA \\
\hline Serviço Social - PPGSS & CCBSA \\
\hline Relações Internacionais - PPGRI & CEDUC \\
\hline Profissional em Matemática - PROFMAT & CCBS \\
\hline Ciência e Tecnologia em Saúde - PPGCTS & CCBS \\
\hline Saúde Pública - PPGSP & - \\
\hline 20 Programas de Pós-Graduação & \\
\hline
\end{tabular}

Fonte: Universidade Estadual da Paraíba (2016). * Pós-graduações ligadas à PRPGP.

Através da análise da produção total de artigos científicos (Figura 5), destaca-se a produtividade dos programas de Pós-graduação das áreas de Saúde (PPGO, PPGSP, PPGCF e PPGE) e das Ciências Biológicas (PPGEC e PPGETNO). Programas dessas duas áreas também apresentam distribuição da produção concentrada em poucos docentes, alguns deles responsáveis por mais de 30\% da produção total média do programa.

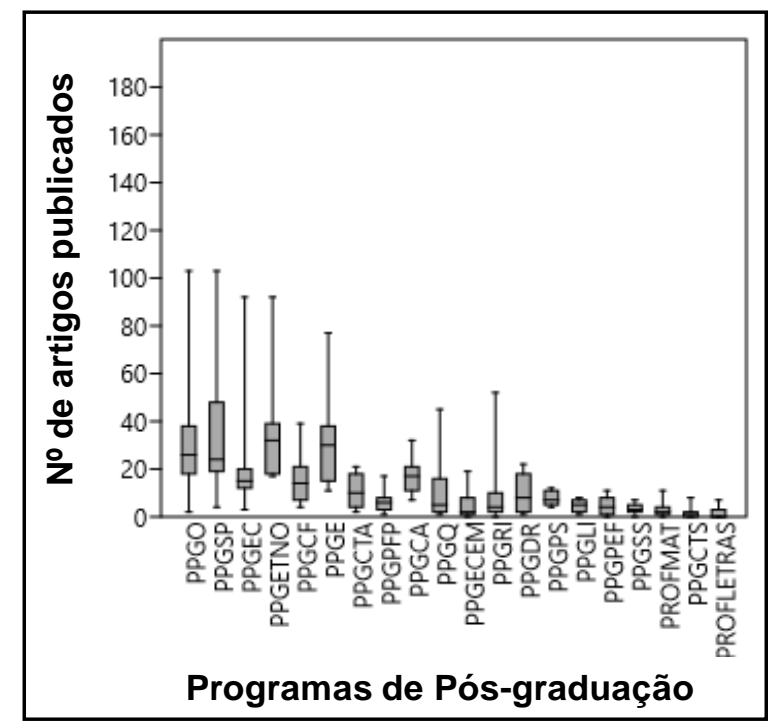

Figura 5. Produção total de artigos periódicos publicados pelos Programas de Pós-graduação. 0 significado das siglas consta na Tabela 4. 
Considerando somente os artigos indexados no SCOPUS e WoS, os docentes com maior produção são àqueles vinculados aos programas de Pós-graduação em Ecologia e Conservação (PPGEC) e Etnobiologia e Conservação da Natureza (PPGETNO) (ver Figura 6). A produção total dos pesquisadores do PPGEC foi de 292 artigos (ver Tabela 6), dos quais $206(70,5 \%)$ estavam indexados ao SCOPUS e $181(62 \%)$ a WoS. Os docentes do PPGETNO produziram 228 artigos. Destes, 158 (69,3\%) estavam indexados à primeira base e $143(62,7 \%)$ à segunda. Comparativamente, os docentes do Programa de PósGraduação em Odontologia (PPGO) publicaram um total de 496 artigos, 180 (36,3\%) dos quais indexados à base SCOPUS e $168(33,9 \%)$ indexados a $W o S$.

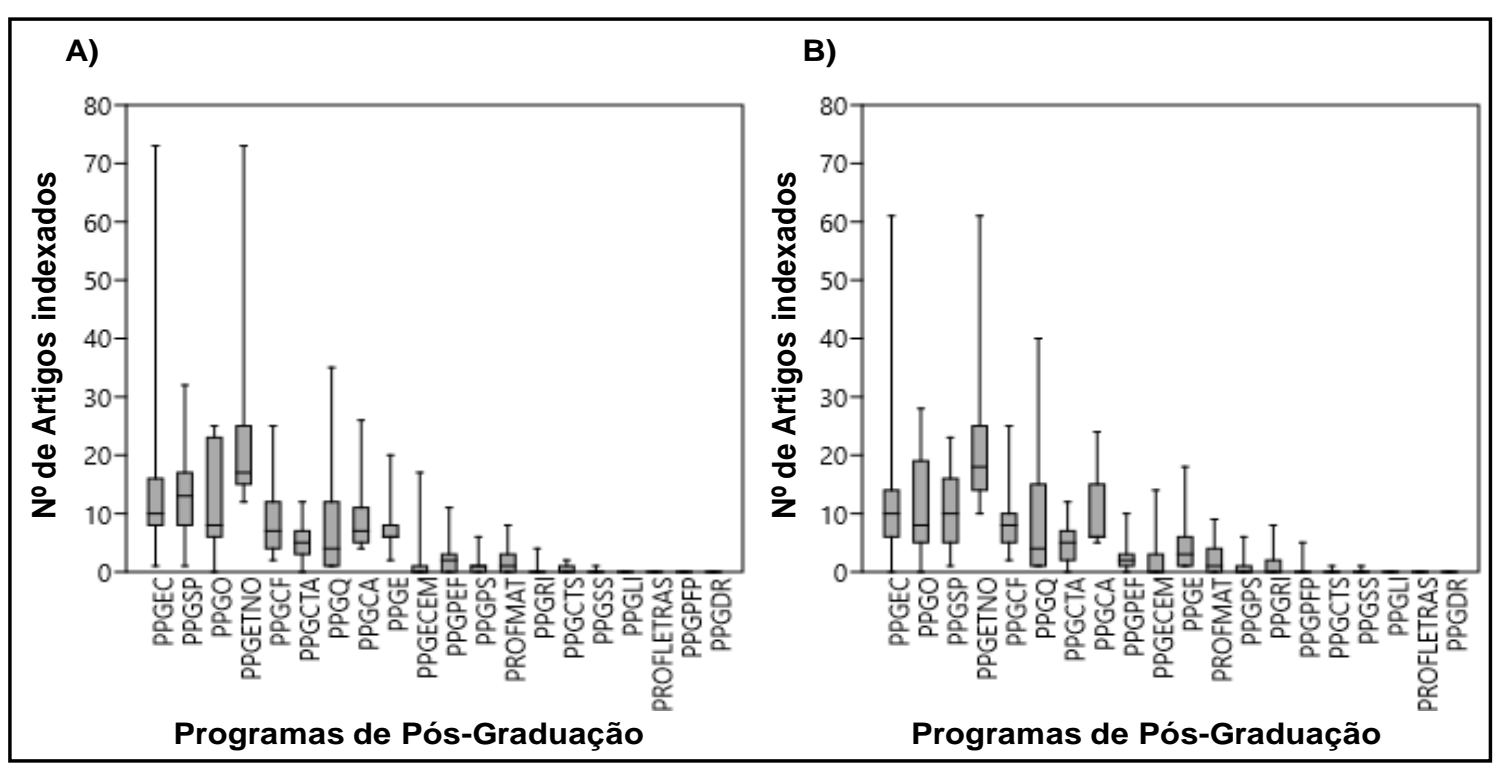

Figura 6. Produção total de artigos periódicos publicados indexados às bases (A) SCOPUS e (B) WoS pelos Programas de Pós-graduação. 0 significado das siglas consta na Tabela 4.

\section{Discussão}

Nossos resultados revelam que a produção científica da Universidade Estadual da Paraíba, no período analisado, está concentrada em uma pequena parcela dos docentes da instituição, muito embora, $76,3 \%$ dos docentes estejam no regime de trabalho T40 DE (Dedicação Exclusiva) à pesquisa e ensino. Forero e Moore (2016) expõe possíveis alternativas que podem refletir positivamente na produtividade científica de um pesquisador. Dentre elas, a necessidade de dedicação exclusiva por parte do docente, determinando um maior empenho e foco nas atividades de pesquisa científica (Bland et al., 2016). No caso da UEPB, apesar do alto número de docentes com dedicação exclusiva, essa condição não se reflete na produção científica da universidade.

Os dados também revelaram que há uma diferença entre as publicações de acordo com as áreas de pesquisa. Algumas delas vêm direcionando sua publicação para capítulos e livros nacionais (Ciências Humanas, Linguística, Letras e Artes e as Ciências Sociais Aplicadas), e outras sendo responsáveis pela maior parte da produção de artigos em periódicos (Ciências da Saúde, Ciências Biológicas, Ciências Exatas e da Terra, Ciências Agrárias, Engenharias e Multidisciplinar). Esses resultados corroboram pesquisas prévias sobre produção científica no Brasil. O trabalho de Silva et al. (2003), por exemplo, 
constatou que a produção científica de pesquisadores nas áreas de Ciências Humanas e Sociais Aplicadas de universidades federais do Brasil é, predominantemente, concentrada em veículos nacionais para a publicação da maioria de suas pesquisas. Estas autoras acreditam que isso se dá pelo fato de uma boa parte dos trabalhos terem discussões acerca de assuntos de interesse regional ou local. Garvey e Griffth (1971) também associa a preferência desses docentes em publicar em livros e capítulos de livros em razão do público-alvo que estes desejam atingir. Tais justificativas se contrapõem ao grande número de revistas científicas que existem em nível internacional, suportada por uma expressiva produção científica de pesquisadores de diversos países do mundo, o que pode ser facilmente constatado através de uma consulta as principais bases de dados acadêmicos, como o SCOPUS e Web of Science, as quais apresentam grande quantidade de artigos das áreas de Ciências Sociais e Humanas publicados.

A análise dos dados da produção científica dos pesquisadores da UEPB não demonstrou diferenças significativas na produção total de acordo com o sexo do docente. Este fato corrobora os resultados da pesquisa de Leta (2003), a qual relata ser mito afirmar que mulheres são menos produtivas do que homens (considerando em seu estudo as subáreas astronomia, imunologia e oceanografia). Este fato também foi observado entre os docentes da UEPB, pois em alguns departamentos, sobretudo àqueles em que há uma maior participação feminina, as docentes mulheres apresentaram uma produtividade científica similar a dos homens. Estes resultados divergem das afirmações dadas por Moschkovich e Almeida (2015), que acreditam que o fato de existirem responsabilidades atribuídas às docentes mulheres, como afazeres domésticos e a maternidade implicam na redução da dedicação exclusiva à pesquisa/trabalho, tornando-as assim, menos produtivas que seus colegas homens. Nossos dados revelam que, pelo menos recentemente e no caso da UEPB, a produção entre pesquisadores não apresenta grande diferença de acordo com o gênero.

Nos dados computados no que se referem à supervisão a discentes de graduação, os maiores números estão relacionados aos docentes das áreas das Ciências Humanas e Ciências Sociais Aplicadas. No entanto, isso não se refletiu em publicações de artigos periódicos, sendo assim, é bem provável que as pesquisas tenham sido divulgadas em canais científicos com pouca visibilidade na comunidade científica (TCC's, dissertações e teses, por exemplo), tornando-se literatura cinza, publicada fora dos canais de publicação e distribuição acadêmicos. No que diz respeito a orientação à discentes de mestrado e doutorado, as áreas das Ciências da Saúde e das Ciências Biológicas apresentaram os maiores percentuais. Além de esses departamentos possuírem um total de oito programas de pós-graduação, vinculados a eles estão 33\% do corpo docente da instituição. Diniz-Filho et al. (2016) mencionam em sua pesquisa a relação positiva entre a alta produção científica do pesquisador à sua participação nos programas de pós-graduação. Herculano e Norberto (2012) também encontraram resultados semelhantes, constatando que os departamentos que possuíam programas de pós-graduação apresentaram as maiores produções científicas.

Os pesquisadores da área de Ciências Biológicas apresentaram a maior média de índice- $h$ quando comparada às outras áreas de conhecimento, indicando que suas pesquisas vêm tendo maior impacto e repercussão em relação a docentes de outras áreas, quando consideramos essa métrica. Naturalmente, também é consequência do fato desses docentes terem grande parte de suas pesquisas publicadas em artigos periódicos indexados às bases internacionais SCOPUS e WoS. No caso da UEPB, podemos associar a ausência ou os baixos índices- $h$ da maioria dos docentes pertencentes à área de Humanas, Ciências Sociais Aplicadas e Linguística, Letras e Artes ao baixo número de publicações de artigos indexados às bases SCOPUS e WoS, embora estas apresentem centenas de periódicos dedicados a pesquisas associadas a tais áreas. 
Herculano e Noberto (2011) em um de seus estudos de avaliação da produção científica do corpo docente da Universidade Estadual Paulista (UNESP), constatou resultados semelhantes ao encontrado no presente estudo no que se refere a produtividade do corpo docente ligado ao departamento de biologia. Foi constatado uma alta produção de artigos científicos indexados às bases SCOPUS e WoS, quando comparada às outras áreas de conhecimento. No caso da UEPB, um dos motivos que pode explicar isso é que o Departamento de Biologia possui vários pesquisadores ligadas à pós-graduação, sobretudo relacionados a grande área de Biodiversidade da CAPES, cuja avaliação da produção está fortemente atrelada a produção de artigos em periódicos com a cobertura por parte das bases internacionais SCOPUS e WoS.

Nos programas de pós-graduação concentram-se os autores responsáveis por alavancar a produção científica das instituições brasileiras. A agência de fomento que avalia esses Programas, a CAPES (Coordenação de Aperfeiçoamento de Pessoal de Nível Superior), trabalha para a expansão e consolidação dos mesmos, e analisa os cientistas e grupos de pesquisa por meio de índices bibliométricos, dando peso maior às publicações em periódicos internacionais de grande impacto (Luiz, 2003). Assim, não surpreende que os docentes vinculados aos programas de pós-graduação da UEPB venham publicando a maior parte dos artigos científicos da instituição, sobretudo considerando a produção indexada às bases SCOPUS e WoS.

Dentre os programas de pós-graduação da UEPB, Odontologia destacou-se pelo maior número de artigos publicados. Essa mesma tendencia tem sido registrada em nível Nacional, uma vez que desde 2006 a produção da área de odontologia vem se destacando quando comparada a outros países (Gonçalves et al. 2019). Segundo estes autores, o Brasil aparece na segunda posição do ranking dos países que mais produziram artigos científicos indexados na área de odontologia, atrás apenas dos Estados Unidos. A posição de destaque do Brasil na pesquisa em odontologia é resultado, em grande parte, da atuação de três universidades estaduais paulistas: Universidade de São Paulo (USP), Universidade Estadual Paulista (UNESP) e Universidade Estadual de Campinas (UNICAMP). Juntas, essas três instituições foram responsáveis por $36,53 \%$ do total de artigos brasileiros publicados em periódicos de odontologia indexados pelo SCOPUS (Lopes, 2019).

No caso da odontologia e alguns outros programas de pós-graduação da UEPB, há de se ponderar a influência de pesquisadores de alta produtividade, via de regra bolsistas de produtividade, que respondem a uma média de $30 \%$ da produção global dos programas de pós. Scarpelli et al. (2008) relata sobre a forte contribuição na produção de artigos por parte de pesquisadores bolsistas PQ vinculados ao campo da Odontologia. Nosso dados revelam, entretanto, que quando se considera a produção destes pesquisadores outliers, parte de suas produções estão relativizadas em publicações não indexadas às bases SCOPUS e WoS, sendo, portanto, parte da produção publicada em revistas regionais e locais não indexadas, com menor incidência em revistas consideradas de melhor qualidade, em nível internacional. Assim, quando consideramos a produção indexada dos programas de pós-graduação, a área das Ciências Biológicas (PPGEC e PPGETNO) apresentaram os maiores percentuais de pesquisas publicadas em artigos periódicos indexados a revistas de circulação internacional. Possivelmente, a pressão imposta aos docentes envolvidos nas pós-graduações por uma produção intelectual de impacto, promove o crescimento da qualidade desses programas e da produtividade do corpo docente vinculados a eles, devendo, no entanto, levar em conta que as áreas adotam diferentes critérios de avaliação da produção, algumas exigindo maior qualidade e visibilidade internacional, outras sendo menos exigente nesse aspecto. 


\section{Conclusão}

Os resultados obtidos nos permitem constatar algumas tendências da produção científica dos docentes da Universidade Estadual da Paraíba. A forma de publicação mais utilizada pelos docentes durante o período analisado (2011-2015) foi artigo em periódico, no entanto a maior parte destes vem sendo publicada pelos docentes vinculados aos programas de pós-graduação e das áreas de Ciências Biológicas e da Saúde. Considerando o período analisado, constata-se também que a maioria dos docentes da UEPB, embora tenha dedicação exclusiva ao ensino e pesquisa (T40 DE), não vem contribuindo de maneira efetiva na produção científica da universidade, que está concentrada em uma minoria de docentes da instituição. Tais dados podem nortear ajustes futuros nas progressões funcionais associadas a carreira docente na instituição. Chama atenção que boa parte dos docentes da UEPB não apresentaram sequer uma publicação no período analisado, e igualmente, que uma pequena parte sequer ter cadastro na Plataforma Lattes.

Os maiores valores de índice-h foram obtidos pelos docentes dos departamentos de Biologia e Enfermagem, sugerindo que as pesquisas publicadas por esses docentes vêm tendo maior impacto em comparação com docentes de outras áreas. As áreas das Ciências Sociais, Ciências Humanas e da Linguística, Letras e Artes foram as responsáveis pelo maior número de publicações de livros e capítulos de livros nacionais (apontando para uma produção local e sem repercussão internacional), sendo muito baixa a publicação de pesquisas no formato de artigos científicos, que quando publicados, geralmente estas não são indexados às bases de internacionais SCOPUS e WoS.

De um modo geral, constatou-se que a produção científica da UEPB está relacionada a docentes de algumas áreas específicas e ligados à pós-graduação. Essa concentração é ainda maior quando se considera a produção de artigos de alto impacto internacional. Constata-se, portanto, que a produção da UEPB poderia ser fortemente incrementada se houvesse uma participação de seu corpo docente de maneira mais homogênea, com participação de um maior número de docentes. Percebe-se ainda a importância das pós-graduações na produção cientifica da instituição, sendo os docentes destas os principais responsáveis pela produção científica em quantidade e qualidade, quando se considera métricas internacionais, consideradas fundamentais para a visibilidade internacional da instituição.

\section{Conflito de interesses}

Os autores declaram não haver conflito de interesses.

\section{Referências}

Abramo, G.; D'Angelo, C. A. Evaluating university research: Same performance indicator, different rankings. Journal of Informetrics, v. 9, p. 514-525, 2015. https://doi.org/ 10.1016/j.joi.2015.04.002

Bland, C. J.; Center, B. A.; Finstad, D. A.; Risbey, K. R.; Staples, J. The impact of appointment type on the productivity and commitment of full-time faculty in research and doctoral institutions. The Journal of Higher Education, v. 77, p. 89-123, 2006. https://doi.org/ 10.1080/00221546.2006.11778920

CAPES - Coordenação de Aperfeiçoamento de Pessoal de Nível Superior. Tabelas de áreas de conhecimento/avaliação. 2017. Disponível em: <http://www.capes.gov.br/avaliacao/ instrumentos-de-apoio/tabela-de-areas-do-conhecimento-avaliacao $>$. Acesso em: 02 fev. 2017. 
Carayol, N.; Matt, M. Individual and collective determinants of academic scientists' productivity. Information Economics and Policy, v. 18, p. 55-72, 2006. https://doi.org/10.1016/j.infoecopol.2005.09.002

Diniz-Filho, J. A. F.; Fioravanti, M. C. S.; Bini, L. M.; Rangel, T. F. Drivers of academic performance in a Brazilian university under a government-restructuring program. Journal of Informetrics, v. 10, p. 151-161, 2016. https://doi.org/10.1016/j.joi.2015.12.004

Forero, D. A.; Moore, J. H. Considerations for higher efficiency and productivity in research activities. BioData Mining, v. 9, n. 35, article number 35, 2016. https://doi.org/10.1186/ s13040-016-0115-3

Garvey, W. D.; Griffith, B. C. Scientific communication: Its role in the conduct of research and creation of knowledge. American Psychologist, v. 26, p. 349-362, 1971. https://psycnet.apa.org/doi/10.1037/h0032059

Gonçalves, A. P. R.; Porto, B. L.; Rodolfo, B.; Faggion Jr, C. M.; Agostini, B. A.; Sousa-Neto, M. D.; Moraes, R. R. Brazilian articles in top-tier dental journals and influence of international collaboration on citation rates. Brazilian Dental Journal, v. 30, p. 307-316, 2019. https://doi.org/10.1590/0103-6440201902826

Hammer, O.; Harper, D. A. T.; Ryan, P. D. PAST: Paleontological Statistics Software Package for Education and Data Analysis. Palaeontologia Electronica, v. 4, n. 1, p. 1-9. 2001.

Herculano, R. D.; Norberto, A. M. Q. Uma análise percentual dos periódicos publicados pelos docentes de fonoaudiologia da Universidade São Paulo. Medicina, v. 42, p. 372-378, 2009. https://doi.org/10.11606/issn.2176-7262.v42i3p372-378

Herculano, R. D.; Norberto, A. M. Q. Produtividade Científica dos docentes da Universidade Estadual Paulista, câmpus de Assis/SP. Medicina, v. 44, p. 249-259, 2011. https://doi.org/10.11606/issn.2176-7262.v44i3p249-259

Herculano, R. D.; Norberto, A. M. Q. Análise da produtividade científica dos docentes da Universidade Estadual Paulista, câmpus de Marília/SP. Perspectivas em Ciência da Informação, v. 17, n. 2, p. 57-70, 2012.

Hirsch, J. E. An index to quantify an individual's scientific research output. Proceedings of the National Academy of Sciences of the United States of America, v. 102, p. 16569-16572, 2005. https://doi.org/10.1073/pnas.0507655102

Leta, J.; Lewison, G. The contribution of women in Brazilian science: A case study in Astronomy, Immunology and Oceanography. Scientometrics, v. 57, p. 339-353, 2003. https://doi.org/10.1023/A:1025000600840

Lopes, C. Pós-graduação stricto sensu e produção científica no Brasil. Brasília: Câmara dos Deputados, Consultoria Legislativa, 2019.

Luiz, A. J. B. Importância de textos em português para a apropriação do conhecimento em ciência e tecnologia no Brasil. Cadernos de Ciência \& Tecnologia, v. 20, p. 543-554, 2003. https://doi.org/10.35977/0104-1096.cct2003.v20.8756

Moschkovich, M.; Almeida, A. M. F. Desigualdades de gênero na carreira acadêmica no Brasil. Dados, v. 58, p. 749-789, 2015. https://doi.org/10.1590/00115258201558

Plataforma Lattes. 2016. Disponível em: <http://buscatextual.cnpq.br/buscatextual/ index.jsp>. Acesso em: 07 out. 2016.

Scarpelli, A. C.; Sardenberg, F.; Goursand, D.; Paiva, S. M.; Pordeus, I. A. Academic trajectories of dental researchers receiving CNPq's productivity grants. Brazilian Dental Journal, v. 19, p. 252-256, 2008. https://doi.org/10.1590/S0103-64402008000300014 
Scopus. Scopus-Elsevier. 2016. Disponível em: <https://www.scopus.com.ez121. periodicos.capes.gov.br/home.uri>. Acesso em: 15 out. 2016.

Silva, E. L.; Menezes. E. M.; Pinheiro, L. V. Avaliação da produtividade científica dos pesquisadores nas Áreas de Ciências Humanas e Sociais Aplicadas. Informação \& Sociedade: Estudos, v. 13, p. 193-222, 2003.

UEPB - Universidade Estadual da Paraíba. Cursos de Graduação. 2016. Disponível em: $<$ http://proreitorias.uepb.edu.br/prograd/cursos-de-graduacao/>. Acesso em: $02 \mathrm{dez}$. 2016.

Vessuri, H. M. C. La revista científica periférica: el caso de Acta Científica Venezolana. Interciencia, v. 12, p. 124-34, 1987.

Velho, L. A ciência e seu público. TransInformação, v. 9, n. 3, p. 15-32, 1997.

Web of Science. Thompson ISI Web of Science. 2016. Disponível em: <http://appswebofknowledge.ez121.periodicos.capes.gov.br/WOS_GeneralSearch_input.do?product= WOS\&search_mode=GeneralSearch\&SID=4ENNEBi9KFblEpgLpum\&preferencesSaved=>. Acesso em: 15 out. 2016. 